\title{
ALTERAÇÕES VOCAIS E CUIDADOS DE SAÚDE ENTRE PROFESSORAS
}

\section{Voice problems and health care among teachers}

\author{
Adriane Mesquita de Medeiros ${ }^{(1)}$, Ada Ávila Assunção ${ }^{(2)}$, Sandhi Maria Barreto ${ }^{(3)}$
}

\begin{abstract}
RESUMO
Objetivo: conhecer os fatores associados à busca de assistência vocal entre professoras. Método: participaram 1.980 professoras do ensino fundamental diurno de 76 escolas públicas. Os dados foram coletados entre maio de 2004 e julho de 2005 por meio de questionário. A variável dependente referiu-se a questão "Nas duas últimas semanas, você procurou médico/fonoaudiólogo por causa de problemas na voz?" As variáveis independentes foram: sintomas de cansaço vocal e de piora na qualidade vocal, aspectos sociodemográficos, aspectos de saúde, percepção de ruído na sala de aula e na escola, ausência e afastamento do trabalho por problemas de voz, trabalho em outra atividade com uso intensivo da voz. A regressão logística foi utilizada para a análise das associações entre a variável dependente e os fatores de interesse com magnitudes estimadas usando-se o odds ratio, com intervalo de confiança de $95 \%$. Resultados: apenas 7\% das professoras buscaram assistência no período de 15 dias. A procura por assistência foi associada ao ruído elevado na sala de aula, relato de piora na voz, problemas de vias aéreas superiores e ausência do trabalho por causa da voz. $O$ presente trabalho mostrou que o comportamento recente é precipitado pela piora ou superposição de problemas vocais e respiratórios. Conclusão: a procura ou não pela assistência à saúde articula-se a fatores relacionados a outras dimensões além do adoecimento. Portanto, questões referentes ao uso de serviços de saúde como um processo dinâmico e relacionado ao distúrbio da voz devem ser abordadas em pesquisas futuras.
\end{abstract}

DESCRITORES: Distúrbios da Voz; Docentes; Assistência à Saúde

\section{INTRODUÇÃO}

Os docentes, quando comparados aos outros grupos populacionais, buscam os serviços de otorrinolaringologia ou fonoaudiologia com menos frequência do que o esperado ${ }^{1}$, se levada em conta a morbidade vocal registrada no setor educacional.

(1) Fonoaudióloga; Professora no Centro Universitário de Belo Horizonte, UNI-BH, Belo Horizonte, Minas Gerais, Brasil; Doutoranda no Programa de Pós-Graduação em Saúde Pública da Universidade Federal de Minas Gerais; Mestre em Saúde Pública, UFMG, Belo Horizonte, Minas Gerais, Brasil.

(2) Médica; Professora na Universidade Federal de Minas Gerais, UFMG, Belo Horizonte, Minas Gerais, Brasil; Pósdoutora em Saúde Pública pela FIOCRUZ, Rio de Janeiro, Rio de Janeiro, Brasil.

(3) Médica; Professora na Universidade Federal de Minas Gerais, UFMG, Belo Horizonte, Minas Gerais, Brasil; Pósdoutora em Saúde Pública, Department of Chronic Diseases and Health Promotion (CHP), WHO, Genebra, Suíça.

Conflito de interesses: inexistente
A diferença entre as taxas de adoecimento e a busca de assistência traz inquietações no campo da saúde pública, porque os sujeitos com alterações vocais estão sob maior risco de afastamento das atividades laborais e de limitações para o desempenho profissional ${ }^{2}$.

Sabe-se também que a busca pela assistência tende a ocorrer em estágios avançados do problema $^{3}$ com implicações diretas sobre o sucesso da reabilitação vocal. O que explicaria esse comportamento? Lacunas e falhas na estruturação dos serviços explicariam a cronicidade dos quadros clínicos que chegam aos serviços, como se vê nos casos de disfonia associada à laringopatia?

Estudo recente realizado em Salvador (Brasil) evidencia a baixa oferta de serviços fonoaudiológicos no SUS e a orientação predominante especializada na estruturação dos serviços fonoaudiológicos, em geral, independentemente do caráter público ou privado da assistência ${ }^{4}$. 
Implicações negativas para a qualidade de vida e para os objetivos de ensino que orientam as atividades do referido grupo ocupacional justificam investigações destinadas a compreender os fatores associados aos comportamentos de proteção à saúde vocal ${ }^{5}$. Conhecer os fatores associados aos cuidados de saúde dos sujeitos reforçaria o paradigma da promoção à saúde que enfrenta as debilidades do diagnóstico tardio e fraquezas da reabilitação vocal nessas situações.

O objetivo deste estudo foi conhecer os fatores associados à busca de assistência médica e fonoaudiológica por causa da voz entre professoras do ensino fundamental.

\section{MÉTODO}

Foi realizado um inquérito sobre as condições de saúde e de trabalho dos professores do ensino fundamental da Rede Municipal de Ensino de Belo Horizonte (RMEBH). Participaram desta análise 1.980 professoras do ensino fundamental diurno de 76 escolas. As professoras de educação física não participaram deste estudo porque realizam atividades de ensino com características bastante diferenciadas das atividades docentes tradicionais. Foram excluídos os homens, dado ao número reduzido de professores encontrado no ensino fundamental da RMEBH, e as professoras que não exerciam a docência na escola ou estavam licenciadas.

A amostra aleatória simples considerou as escolas municipais que estavam em funcionamento em 2004, nos turnos da manhã e tarde. Foram sorteadas $70 \%$ das escolas com representatividade de no mínimo $80 \%$ das professoras. A coleta de dados ocorreu entre maio de 2004 e julho de 2005 por meio de um questionário auto-aplicado, organizado especialmente para esta pesquisa.

A variável dependente referiu-se a questão "Nas duas últimas semanas, você procurou médico / fonoaudiólogo por causa de problemas na voz? (respostas: não/sim)". As variáveis independentes foram organizadas em blocos, para a entrada hierarquizada no modelo de análise: a) Características sociodemográficas: idade, escolaridade, estado civil, filhos, renda pessoal e renda total familiar; b) Aspectos da saúde: uso de medicamentos para depressão e para alteração do sono, depressão/ansiedade (General Health Questionnaire-12 /GHQ-12), infecções de vias aéreas superiores, hidratação durante a aula, tabagismo, prática de atividade física; c) Características ocupacionais: tempo de docência, número de turnos que leciona, outro trabalho com uso intensivo da voz, relacionamento com superiores e colegas, percepção de ruído na sala de aula e na escola, ventilação da sala e condições da parede, possibilidade de ser criativo, margem de autonomia, tempo para preparar aula e para corrigir trabalhos; d) Aspectos relacionados à voz: sintomas de cansaço vocal e de piora na qualidade vocal nos últimos 15 dias, problemas no trabalho por causa da voz, falta e afastamento do trabalho por problemas de voz.

O projeto desta pesquisa foi aprovado pelo Comitê de Ética em Pesquisa local em 05 de outubro de 2004 (Parecer no 240) e todos os participantes assinaram o termo de consentimento livre e esclarecido.

A regressão logística múltipla foi utilizada para identificar os fatores associados à procura por médico e/ou fonoaudiólogo por causa da voz nos últimos 15 dias. As magnitudes das associações entre a variável dependente e os fatores de interesse foram estimadas usando-se o odds ratio (OR), com intervalo de confiança de 95\% (IC95\%).

Todos os fatores associados ao nível de $p \leq 0,20$ na análise univariada foram incluídos no modelo multivariado por blocos de afinidade das variáveis, sendo retidos os fatores que permaneceram associados em nível $p \leq 0,05$ para construção do modelo final. A análise utilizou programa STATA, versão 8.0 (Stata Corp., College Station, Estados Unidos).

\section{RESULTADOS}

$\mathrm{Na}$ análise univariada, não houve associação entre a procura por assistência vocal e as características sociodemográficas (TABELA 1). Em relação à saúde geral das professoras, o uso de medicamento para o sono, presença de depressão/ ansiedade (15 dias), problemas de vias aéreas superiores (15 dias) e o hábito de hidratação durante as aulas foram associados à variável dependente (TABELA 2). Entre as características ocupacionais, houve associação significante com a presença de ruído elevado a insuportável na sala de aula e na escola, pequena autonomia e pouco tempo para correção de trabalhos (TABELA 3). Todos os aspectos relacionados à voz - cansaço ao falar (15 dias), piora na qualidade da voz (15 dias), problemas no trabalho por causa da voz (15 dias), falta no trabalho por causa da voz ( 15 dias), afastamento no trabalho por causa da voz - apresentaram associação estatisticamente significante (TABELA 4).

No modelo final, mantiveram-se significantes as variáveis: problemas de vias aéreas superiores, presença de ruído elevado a insuportável na sala de aula, percepção de piora na qualidade vocal e faltar ao trabalho por causa da voz (TABELA 5). 
Tabela 1 - Análise univariada da associação entre as características sociodemográficas e a procura pela assistência vocal

\begin{tabular}{|c|c|c|c|c|}
\hline \multirow[t]{2}{*}{ Fatores } & \multicolumn{2}{|c|}{$\begin{array}{l}\text { Procurou médico/fono por } \\
\text { causa da voz }\end{array}$} & \multirow[t]{2}{*}{$\mathrm{OR}^{\star}(\mathrm{IC} 95 \%)^{\star \star}$} & \multirow[t]{2}{*}{$\mathbf{p}^{\star \star *}$} \\
\hline & Não n (\%) & Sim n (\%) & & \\
\hline \multicolumn{5}{|l|}{ Idade (anos) } \\
\hline $19-29$ & $104(5,67)$ & $11(8,09)$ & 1,0 & 0,69 \\
\hline $30-39$ & $590(32,17)$ & $40(29,41)$ & $0,64(0,32-1,29)$ & \\
\hline $40-49$ & $768(41,88)$ & $57(41,91)$ & $0,70(0,36-1,38)$ & \\
\hline $50-67$ & $372(20,28)$ & $28(20,59)$ & $0,71(0,34-1,48)$ & \\
\hline \multicolumn{5}{|l|}{ Escolaridade } \\
\hline Pós-graduação & $911(50,05)$ & $71(52,21)$ & 1,0 & 0,18 \\
\hline Superior & $790(43,41)$ & $61(44,85)$ & $0,99(0,69-1,41)$ & \\
\hline Médio & $119(6,54)$ & $4(2,94)$ & $0,43(0,15-1,20)$ & \\
\hline \multicolumn{5}{|l|}{ Estado civil } \\
\hline Solteiro, separado, divorciado ou viúvo & $1,072(58,64)$ & $71(52,21)$ & 1,0 & 1,14 \\
\hline Casado ou união consensual & $756(41,36)$ & $65(47,79)$ & $1,30(0,92-1,84)$ & \\
\hline \multicolumn{5}{|l|}{ Filhos } \\
\hline 0 & $504(28,88)$ & $41(32,54)$ & 1,0 & 0,62 \\
\hline $1-2$ & $922(52,84)$ & $65(51,59)$ & $0,87(0,58-1,30)$ & \\
\hline $3-8$ & $319(18,28)$ & $20(15,87)$ & $0,77(0,44-1,34)$ & \\
\hline \multicolumn{5}{|l|}{ Renda total pessoal } \\
\hline Mais de $\mathrm{R} \$ 2400,00$ & $281(15,45)$ & $25(18,38)$ & 1,0 & 0,28 \\
\hline$R \$ 1600,00-R \$ 2400,00$ & $704(38,70)$ & $58(42,65)$ & $0,93(0,57-1,51)$ & \\
\hline$R \$ 240,00-R \$ 1600,00$ & $834(45,85)$ & $53(38,97)$ & $0,71(0,44-1,17)$ & \\
\hline \multicolumn{5}{|l|}{ Renda total familiar } \\
\hline Mais de $R \$ 2400,00$ & $887(48,92)$ & $69(51,11)$ & 1,0 & 0,88 \\
\hline$R \$ 1600,00-R \$ 2400,00$ & $623(34,36)$ & $45(33,33)$ & $0,93(0,63-1,37)$ & \\
\hline $\mathrm{R} \$ 240,00-\mathrm{R} \$ 1600,00$ & $303(16,71)$ & $21(15,56)$ & $0,89(0,54-1,48)$ & \\
\hline
\end{tabular}

\section{DISCUSSÃO}

Apenas $7 \%$ das professoras buscaram assistência relacionada à voz no período de 15 dias que antecederam a coleta de dados. Após o ajuste entre as variáveis estatisticamente significativas na análise univariada, a procura por assistência vocal manteve-se associada ao ruído elevado a insuportável na sala de aula, relato de piora na qualidade vocal, problemas de vias aéreas superiores e ausência ao trabalho por causa da voz.

O ruído elevado a insuportável gerado na sala de aula foi o único fator ambiental que permaneceu no modelo. $O$ estudo de Chen et al. ${ }^{2}$ encontrou que o uso intenso da voz é o principal fator de risco para desenvolver o distúrbio vocal em professores. Sabe-se que os professores tendem a desenvolver ajustes negativos, como falar em forte intensidade e com esforço quando expostos ao ruído por longos períodos e sem intervalo para repouso ou recuperação'. É plausível supor que a dificuldade de se sobrepor ao ruído da escola, principalmente da sala (de acordo com a percepção do respondente) tenha relação com a busca pela assistência vocal.

A associação encontrada entre piora da voz e busca recente de assistência vocal não seria inesperado. Entretanto, é surpreendente que o sintoma de cansaço vocal, muito prevalente entre as professoras $^{6}$, não se manteve associado ao desfecho de interesse. Pode ser que o sintoma per si não mobilize o sujeito ou não esteja representado como um problema, ao se considerar a afirmativa de Bazzo e Noronha ${ }^{4}$ sobre as razões do acesso aos serviços. 
Tabela 2 - Análise univariada da associação entre aspectos da saúde e a procura pela assistência vocal

\begin{tabular}{|c|c|c|c|c|}
\hline \multirow[t]{2}{*}{ Fatores } & \multicolumn{2}{|c|}{$\begin{array}{l}\text { Procurou médico/fono por } \\
\text { causa da voz }\end{array}$} & \multirow[t]{2}{*}{$\mathrm{OR}^{\star}(\mathrm{IC} 95 \%)^{\star \star}$} & \multirow[t]{2}{*}{$\mathbf{p}^{\star \star *}$} \\
\hline & Não n (\%) & Sim n (\%) & & \\
\hline \multicolumn{5}{|c|}{ Uso de medicamento para depressão } \\
\hline Não & $1.408(76,77)$ & $99(72,79)$ & 1,0 & 0,30 \\
\hline Sim & $426(23,23)$ & $37(27,21)$ & $1,24(0,83-1,83)$ & \\
\hline \multicolumn{5}{|c|}{$\begin{array}{l}\text { Uso de medicamento para alteração } \\
\text { do sono }\end{array}$} \\
\hline Não & $1.646(89,75)$ & $110(80,88)$ & 1,0 & $<0,01$ \\
\hline Sim & $188(10,25)$ & $26(19,12)$ & $2,07(1,32-3,26)$ & \\
\hline \multicolumn{5}{|l|}{ Depressão/ ansiedade (GHQ) } \\
\hline Não & $924(50,38)$ & $40(29,41)$ & 1,0 & $<0,01$ \\
\hline Sim & $910(49,62)$ & $96(70,59)$ & $2,44(1,67-3,56)$ & \\
\hline \multicolumn{5}{|c|}{$\begin{array}{l}\text { Presença de processo inflamatório, } \\
\text { infeccioso ou alérgico de vias aéreas } \\
\text { superiores (últimos } 15 \text { dias) }\end{array}$} \\
\hline Não & $1.094(59,95)$ & $37(27,21)$ & 1,0 & $<0,01$ \\
\hline $\operatorname{Sim}$ & $731(40,05)$ & $99(72,79)$ & $4(2,71-5,91)$ & \\
\hline \multicolumn{5}{|l|}{ Hidratação durante a aula } \\
\hline Não & $518(28,51)$ & $25(18,52)$ & 1,0 & 0,01 \\
\hline Sim & $1.299(71,49)$ & $110(81,48)$ & $1,76(1,12-2,74)$ & \\
\hline \multicolumn{5}{|l|}{ Tabagismo } \\
\hline Não & $1.331(74,44)$ & $90(68,18)$ & 1,0 & 0,11 \\
\hline Sim & $457(25,56)$ & $42(31,82)$ & $1,36(0,93-1,99)$ & \\
\hline \multicolumn{5}{|l|}{ Atividade física regular } \\
\hline 3 ou mais vezes por semana & $391(21,64)$ & $31(23,31)$ & 1,0 & 0,90 \\
\hline $1-2$ vezes por semana & $563(31,16)$ & $40(30,08)$ & $0,90(0,55-1,46)$ & \\
\hline Nenhuma vez & $853(47,21)$ & $62(46,62)$ & $0,92(0,59-1,43)$ & \\
\hline
\end{tabular}

Vianello et al. ${ }^{7}$ sugerem que a vigência da voz alterada é tratada como algo inexorável à profissão. Comportamentos deste gênero ou estratégias de compensação dos déficits explicariam porque os sujeitos da amostra buscaram assistência apenas quando perceberam agravamento dos sintomas vocais. Na análise univariada, encontrou-se que as professoras que de procuram assistência vocal relataram problemas moderados ou freqüentes no trabalho por causa da voz e realizaram a hidratação durante as aulas. A hidratação adequada é amplamente divulgada em ações de promoção da saúde vocal e de reabilitação e provavelmente adotada por aquelas que já apresentam sintomas vocais.

Observou-se também que as professoras que procuram assistência vocal, comparadas às que não procuram, relataram pequena margem de autonomia e pouco tempo para corrigir os trabaIhos, sem considerar os demais aspectos investigados. O presente resultado é indicador da possível influência da organização do trabalho escolar na decisão do professor em procurar ajuda por causa da voz.

A associação entre busca de atenção a saúde, problemas de vias aéreas superiores e falta ao trabalho nas duas semanas anteriores é esperada e sugere precipitação do quadro associada à agudização ou superposição de queixas. Apesar de não permanecer no modelo final, as professoras que procuram assistência quando comparadas àquelas que não o fizeram têm mais chance de apresentar problemas de depressão e ansiedade e o uso de 
Tabela 3 - Análise univariada da associação entre características ocupacionais e a procura pela assistência vocal

\begin{tabular}{|c|c|c|c|c|}
\hline \multirow[t]{2}{*}{ Fatores } & \multicolumn{2}{|c|}{$\begin{array}{l}\text { Procurou médico/fono por } \\
\text { causa da voz }\end{array}$} & \multirow[t]{2}{*}{ OR $^{\star}(\text { IC } 95 \%)^{\star \star}$} & \multirow[t]{2}{*}{$\mathbf{p}^{\star \star *}$} \\
\hline & Não n (\%) & Sim n (\%) & & \\
\hline \multicolumn{5}{|c|}{ Tempo de docência (anos) } \\
\hline $0-4$ & $45(5,64)$ & $2(3,45)$ & 1,0 & 0,41 \\
\hline $5-9$ & $61(7,64)$ & $3(5,17)$ & $1,11(0,18-6,90)$ & \\
\hline $10-14$ & $138(17,29)$ & $13(22,41)$ & $2,12(0,46-9,75)$ & \\
\hline $15-19$ & $186(23,31)$ & $9(15,52)$ & $1,09(0,23-5,21)$ & \\
\hline$\geq 20$ & $368(46,12)$ & $31(53,45)$ & $1,90(0,44-8,20)$ & \\
\hline \multicolumn{5}{|c|}{ Número de turnos em que leciona } \\
\hline 1 & $595(32,80)$ & $41(30,83)$ & 1,0 & 0,87 \\
\hline $2-3$ & $1.219(67,20)$ & $92(69,17)$ & $1,10(0,75-1,61)$ & \\
\hline \multicolumn{5}{|c|}{$\begin{array}{l}\text { Outro trabalho com uso intensivo } \\
\text { da voz }\end{array}$} \\
\hline Não & $1.514(82,96)$ & $117(86,67)$ & 1,0 & 0,25 \\
\hline Sim & $311(17,04)$ & $18(13,33)$ & $0,75(0,45-1,25)$ & \\
\hline \multicolumn{5}{|c|}{ Relacionamento com superiores } \\
\hline Bom & $1451(80,08)$ & $103(76,30)$ & 1,0 & 0,16 \\
\hline Razoável & $254(14,02)$ & $18(13,33)$ & $1,0(0,59-1,68)$ & \\
\hline Ruim & $107(5,91)$ & $14(10,37)$ & $1,84(1,02-3,33)$ & \\
\hline \multicolumn{5}{|c|}{$\begin{array}{l}\text { Relacionamento com colegas } \\
\text { de trabalho }\end{array}$} \\
\hline Bom & $1556(85,78)$ & $116(85,93)$ & 1,0 & 0,99 \\
\hline Razoável & $206(11,36)$ & $15(11,11)$ & $0,98(0,56-1,70)$ & \\
\hline Ruim & $52(2,87)$ & $4(2,96)$ & $1,03(0,37-2,90)$ & \\
\hline \multicolumn{5}{|c|}{ Ruído gerado na sala de aula } \\
\hline Desprezível a razoável & $932(51,04)$ & $39(28,89)$ & 1,0 & $<0,01$ \\
\hline Elevado a insuportável & $894(48,96)$ & $96(71,11)$ & $2,57(1,75-3,76)$ & \\
\hline \multicolumn{5}{|l|}{ Ruído gerado na escola } \\
\hline Desprezível a razoável & $953(52,25)$ & $57(42,22)$ & 1,0 & 0,02 \\
\hline Elevado a insuportável & $871(47,75)$ & $78(57,78)$ & $1,50(1,05-2,13)$ & \\
\hline \multicolumn{5}{|c|}{ Ventilação na sala de aula } \\
\hline Satisfatória & $566(31)$ & $36(26,67)$ & 1,0 & 0,57 \\
\hline Razoável & $805(44,09)$ & $64(47,41)$ & $1,25(0,82-1,91)$ & \\
\hline Precária & $455(24,92)$ & $35(25,93)$ & $1,21(0,75-1,96)$ & \\
\hline \multicolumn{5}{|c|}{ Condições das paredes da sala de aula } \\
\hline Satisfatória & $926(50,68)$ & $59(43,70)$ & 1,0 & 0,29 \\
\hline Razoável & $765(41,87)$ & $65(48,15)$ & $1,33(0,93-1,92)$ & \\
\hline Precária & $136(7,44)$ & $11(8,15)$ & $1,27(0,65-2,48)$ & \\
\hline \multicolumn{5}{|c|}{ Possibilidade de ser criativo } \\
\hline Grande & $882(48,33)$ & $59(43,38)$ & 1,0 & 0,19 \\
\hline Razoável & $739(40,49)$ & $55(40,44)$ & $1,11(0,76-1,63)$ & \\
\hline Pequena & $204(11,18)$ & $22(16,18)$ & $1,61(0,97-2,69)$ & \\
\hline \multicolumn{5}{|l|}{ Margem de autonomia } \\
\hline Grande & $489(26,87)$ & $33(24,44)$ & 1,0 & 0,03 \\
\hline Razoável & $980(53,85)$ & $63(46,67)$ & $0,95(0,62-1,47)$ & \\
\hline Pequena & $351(19,29)$ & $39(28,89)$ & $1,65(1,02-2,67)$ & \\
\hline \multicolumn{5}{|c|}{ Tempo para preparação de aulas } \\
\hline Grande & $342(18,98)$ & $20(14,81)$ & 1,0 & 0,31 \\
\hline Razoável & $828(45,95)$ & $60(44,44)$ & $1,24(0,74-2,09)$ & \\
\hline Pequeno & $635(35,07)$ & $55(40,74)$ & $1,49(0,88-2,52)$ & \\
\hline \multicolumn{5}{|c|}{ Tempo para correção de trabalhos } \\
\hline Grande & $277(15,42)$ & $11(8,15)$ & 1,0 & 0,02 \\
\hline Razoável & $744(41,43)$ & $52(38,52)$ & $1,76(0,91-3,42)$ & \\
\hline Pequeno & $775(43,15)$ & $72(53,33)$ & $2,34(1,22-4,48)$ & \\
\hline
\end{tabular}


Tabela 4 - Análise univariada da associação entre aspectos relacionados à voz e a procura pela assistência vocal

\begin{tabular}{|c|c|c|c|c|}
\hline \multirow[t]{2}{*}{ Fatores } & \multicolumn{2}{|c|}{$\begin{array}{l}\text { Procurou médico/fono } \\
\text { por causa da voz }\end{array}$} & \multirow[t]{2}{*}{$\mathrm{OR}^{*}(\mathrm{IC} 95 \%)^{\star *}$} & \multirow[t]{2}{*}{$\mathbf{p}^{\star \star \star}$} \\
\hline & Não n (\%) & Sim n (\%) & & \\
\hline \multicolumn{5}{|c|}{ Cansaço ao falar (15 dias) } \\
\hline Não & $763(41,85)$ & $9(6,67)$ & 1,0 & $<0,01$ \\
\hline Ás vezes & $872(47,83)$ & $72(53,33)$ & $7(3,48-14,09)$ & \\
\hline Diariamente & $188(10,31)$ & $54(40)$ & $24,35(11,81-50,20)$ & \\
\hline \multicolumn{5}{|c|}{ Piora da qualidade vocal (15 dias) } \\
\hline Não & $847(46,44)$ & $6(4,44)$ & 1,0 & $<0,01$ \\
\hline Ás vezes & $803(44,02)$ & $66(48,89)$ & $11,60(5-26,91)$ & \\
\hline Diariamente & $174(9,54)$ & $63(46,67)$ & $51(21,78-119,96)$ & \\
\hline \multicolumn{5}{|c|}{$\begin{array}{l}\text { Problemas no trabalho por causa da voz } \\
\text { (últimos } 15 \text { dias) }\end{array}$} \\
\hline ausente & $1.547(84,95)$ & $68(50,37)$ & 1,0 & $<0,01$ \\
\hline moderado & $187(10,27)$ & $36(26,67)$ & $4,38(2,84-6,74)$ & \\
\hline freqüente & $87(4,78)$ & $31(22,96)$ & $8,11(5,03-13,05)$ & \\
\hline \multicolumn{5}{|c|}{$\begin{array}{l}\text { Faltou ao trabalho por causa da voz } \\
\text { (últimos } 15 \text { dias) }\end{array}$} \\
\hline Não & $1.806(98,58)$ & $96(70,59)$ & 1,0 & $<0,01$ \\
\hline Sim & $26(1,42)$ & $40(29,41)$ & $28,94(16,95-49,41)$ & \\
\hline \multicolumn{5}{|c|}{$\begin{array}{l}\text { Afastamento da carreira por causa } \\
\text { da voz }\end{array}$} \\
\hline Nunca & $1.315(71,98)$ & $66(48,53)$ & 1,0 & $<0,01$ \\
\hline Há mais de 6 meses & $453(24,79)$ & $42(30,88)$ & $1,85(1,24-2,76)$ & \\
\hline Nos últimos seis meses & $59(3,23)$ & $28(20,59)$ & $9,46(5,66-15,80)$ & \\
\hline
\end{tabular}

medicamentos prescritos para alteração do sono. Os professores com distúrbio vocais, no estudo de Chen et al. ${ }^{2}$, tiveram mais chance de problemas de vias aéreas superiores, estresse e ansiedade, quando comparados aos professores sem queixas vocais. Adicionalmente, os sujeitos da amostra apresentaram um maior número de doenças associadas.

A necessidade de faltar ao trabalho por causa da voz associada à procura pelo especialista, além de indicar uma limitação no desempenho da docência, pode ser um indicador da dificuldade de disponibilidade de tempo para buscar assistência. Apesar de não permanecer no modelo final, as professoras que relatam afastamento da carreira por causa da voz são as que mais procuram ajuda.

Vários fatores relacionados às barreiras do acesso e utilização dos serviços de saúde não foram abordados no presente estudo e precisam ser aprofundados. O uso de serviços de saúde pelos professores pode resultar de um processo de interação entre fatores relacionados ao indivíduo, ao sistema de saúde e ao contexto econômicosocial, como enfatiza Andersen ${ }^{8}$. Para o autor, a utilização dos serviços de saúde seria resultado de um processo do tipo cadeia de retroalimentação em que um grupo de fatores influencia de forma dinâmica os fatores predisponentes, a percepção da necessidade de serviços e os comportamentos de saúde.

No quadro do presente estudo, a busca por assistência referiu-se apenas aos últimos 15 dias. Como os problemas vocais são cumulativos com tendência a cronicidade, seria mais vantajoso investigar o processo de busca por assistência no curso do problema e não exclusivamente ao período recente. 
Tabela 5 - Modelo final dos fatores associados à procura pela assistência vocal

\begin{tabular}{|c|c|c|}
\hline \multirow[t]{2}{*}{ Fatores associados } & \multicolumn{2}{|c|}{$\begin{array}{l}\text { Procurou médico/fonoaudiólogo } \\
\text { por causa da voz }\end{array}$} \\
\hline & OR $^{*}$ & IC $(95 \%)^{\star \star}$ \\
\hline \multicolumn{3}{|c|}{ Ruído gerado na sala de aula } \\
\hline Desprezível e razoável & 1,0 & \\
\hline Elevado e insuportável & 1,63 & $1,05-2,52$ \\
\hline \multicolumn{3}{|c|}{$\begin{array}{l}\text { Presença de processo inflamatório, } \\
\text { infeccioso ou alérgico de vias aéreas superiores } \\
\text { (últimos } 15 \text { dias) }\end{array}$} \\
\hline Não & 1,0 & \\
\hline Sim & 1,72 & $1,11-2,68$ \\
\hline \multicolumn{3}{|c|}{$\begin{array}{l}\text { Faltou ao trabalho por causa da voz } \\
\text { (últimos } 15 \text { dias) }\end{array}$} \\
\hline Não & 1,0 & \\
\hline Sim & 15,85 & $8,73-28,81$ \\
\hline \multicolumn{3}{|c|}{ Piora da qualidade vocal (últimos 15 dias) } \\
\hline Não & 1,0 & \\
\hline Ás vezes & 7,79 & $3,29-18,45$ \\
\hline Diariamente & 25,87 & $10,61-63,07$ \\
\hline
\end{tabular}

\section{CONCLUSÃO}

Os resultados do presente estudo mostram que as professoras procuram pela assistência vocal quando apresentam problemas de vias aéreas superiores, diante da percepção de piora na qualidade vocal e na presença de ruído elevado a insuportável na sala de aula, com impacto no desempenho da docência gerado pelo absenteísmo.

A procura ou não pela assistência à saúde articula-se a fatores relacionados a outras dimensões além do adoecimento. Portanto, questões referentes ao uso de serviços de saúde como um processo dinâmico e relacionado ao distúrbio da voz devem ser abordadas em pesquisas futuras.

\section{AGRADECIMENTOS}

A FAPEMIG e a CAPES pelo apoio financeiro a esta pesquisa. 


\begin{abstract}
Purpose: to know factors associated with seeking vocal assistance among teachers. Method: 1,980 day-shift female teachers of 76 primary schools took part in the study. Data were collected between May 2004 and July 2005 using a questionnaire. The dependent variable referred to the question "In the last two weeks, have you sought medical / audiological help because of vocal problems?" The independent variables were: symptoms of vocal fatigue and deterioration in vocal quality, sociodemographic characteristics, health aspects, perceived noise in the classroom and at school, work absenteeism and vocal problems working in other activity using intensive voice. Logistic regression was usein order to analyze associations among the dependent variable and the concerning factors with magnitudes being estimated using the odds ratio with $95 \%$ confidence interval. Results: only $7 \%$ of the teachers sought assistance within 15 days. The demand for care was associated with high noise in the classroom, reporting worsening voice, upper respiratory problems and absence from work because of vocal problems. Conclusion: demand or not for health care is related to factors other than illness. Thus, issues concerning the use of health services as a dynamic process related to vocal disorders must be addressed in future researches.
\end{abstract}

KEYWORDS: Voice Disorders; Faculty; Delivery of Health Care

\section{REFERÊNCIAS}

1. Roy N, Merrill RM, Thibeault S, Parsa RA, Gray $\mathrm{SD}$, Smith EM. Voice disorders in teachers and the general population: effects on work performance, attendance, and future career choices. J Speech Lang Hear Res. 2004; 44:542-52.

2. Chen SH, Chiang SC, Chung YM, Hsiao LC, Hsiao TY. Risk factors and effects of voice problems for teachers. J. Voice. 2010; 24(2): 183-92.

3. Fortes FSG, Imamura R, Tsuji DH, Sennes LU. Perfil dos profissionais da voz com queixas vocais atendidos em um centro terciário de saúde. Rev Bras Otorrinolaringol. 2007;73(1):27-31.

4. Bazzo LMF, Noronha CV. A ótica dos usuários sobre a oferta do atendimento fonoaudiológico no Sistema Único de Saúde (SUS) em Salvador. Cienc. Saude Col. 2009; 14(1):1553-64.

5. Ferreira LP, Servilha EAM, Masson MLV, Reinaldi MBFM. Políticas públicas e voz do professor: caracterização das leis brasileiras. Rev. Soc. Bras. Fonoaudiol. 2009; 14(1):1-7.

6. Araújo TM, Reis EJFB, Carvalho FM, Porto LA, Reis IC, Andrade JM. Fatores associados a alterações vocais em professoras. Cad. Saúde Pública. 2008; 24(6):1229-38.

7. Vianello L, Assunção AA, Gama ACC. Estratégias implementadas para enfrentar as exigências vocais da sala de aula: o caso das professoras readaptadas por disfonia. Distúrb Comum. 2008; 20(2):163-70.

8. Andersen RM. Revisiting the behavorial model and acess to medical care: does it matter? J Health Soc Behav. 1995; 36(1):1-10.

http://dx.doi.org/10.1590/S1516-18462011005000146

RECEBIDO EM: 10/02/2011

ACEITO EM: 07/04/2011

Endereço para correspondência:

Adriane Mesquita de Medeiros

Departamento de Medicina Preventiva e Social

Universidade Federal de Minas Gerais

Av. Alfredo Balena, 190/705

Belo Horizonte, MG, Brasil

CEP: 30310-100

E-mail: adrianemmedeiros@ @otmail.com 